\title{
On numerical solutions of time-fraction generalized Hirota Satsuma coupled KdV equation
}

\author{
Ebru Cavlak Aslan ${ }^{\mathrm{a}}$, Mustafa Inc ${ }^{\mathrm{a}, *}$, Maysaa' Mohamed Al Qurashi $^{\mathrm{b}}$, Dumitru Baleanu ${ }^{\mathrm{c}}$ \\ a Firat University, Science Faculty, Department of Mathematics, 23119, Elazig, Turkey. \\ ${ }^{b}$ Department of Mathematics, King Saud University, P. O. Box 22452, Riyadh, 11495, Saudi Arabia. \\ ${ }^{c}$ Department of Mathematics, Cankaya University, 06530, Balgat, Ankara, Turkey.
}

Communicated by X. J. Yang

\begin{abstract}
In this study, we obtain the approximate soliton solution of the fractional generalized Hirota-Satsuma coupled Kortewegde Vries equation (GHS-cKdV) within the homotopy analysis method (HAM). Numerical results are successfully compared with other solutions obtained by the differential transform method (DTM) and the homotopy perturbation method (HPM). The numerical results indicate that the only few terms are sufficient to get the correct solutions. Also, the results are given by tables and figures. (C)2017 All rights reserved.
\end{abstract}

Keywords: HAM, fractional partial differential equation(FPD), HS-cKdV equation, time-fractional GHS-cKdV equation. 2010 MSC: 34A08, 26A33.

\section{Introduction}

During the past few decades, the fractional equations have been occurred from structures in different fields of science and engineering [20]. The fractional KdV equation and the fractional HSKdV are the most important of these type equations [1, 21, 23]. Firstly, Gardner et al. found analytical solution for cKdV, which describes interactions of two long waves with different dispersion relations $[6,9]$, later Hirota and Satsuma introduced a cKdV equation

$$
\begin{gathered}
\eta_{\vartheta}-a\left(\eta_{\rho \rho \rho}+6 \eta \eta_{\rho}\right)=2 b \varphi \varphi_{\rho} \\
\varphi_{\vartheta}+\varphi_{\rho \rho \rho}+3 \eta \varphi_{\rho}=0
\end{gathered}
$$

where $a$ and $b$ are arbitrary constants [10]. $2 b \varphi \varphi_{\rho}$ acts as a force term on the KdV wave system with the linear dispersion relation $\omega=a k^{3}$. From the equations (1.1) and (1.2), consider the GHS-cKdV equation [1]

\footnotetext{
*Corresponding author

Email addresses: ebrucavlak@hotmail.com (Ebru Cavlak Aslan), minc@firat.edu.tr (Mustafa Inc), maysaa@ksu.edu.sa (Maysaa' Mohamed Al Qurashi), dumitru@cankaya.edu.tr (Dumitru Baleanu)

doi:10.22436/jnsa.010.02.33
} 


$$
\begin{aligned}
& \eta_{\vartheta}=\frac{1}{2} \eta_{\rho \rho \rho}-3 \eta \eta_{\rho}+3(\lambda \varpi)_{\rho}, \\
& \lambda_{\vartheta}=-\lambda_{\rho \rho \rho}+3 \eta \lambda_{\rho}, \quad \varpi_{\vartheta}=-\varpi_{\rho \rho \rho}+3 \eta \varpi_{\rho} .
\end{aligned}
$$

In this study, we consider the solution of GHS-cKdV of time-fractional order

$$
\begin{aligned}
& { }^{{ }^{c}} D_{\vartheta}^{\alpha} \eta=\frac{1}{2} \eta_{\rho \rho \rho}-3 \eta \eta_{\rho}+3(\lambda \varpi)_{\rho}, \\
& { }^{c} D_{\vartheta}^{\alpha} \lambda=-\lambda_{\rho \rho \rho}+3 \eta \lambda_{\rho}, \quad{ }^{c} D_{\vartheta}^{\alpha} \varpi=-\varpi_{\rho \rho \rho}+3 \eta \varpi_{\rho},
\end{aligned}
$$

$0<\alpha<1$. If $\xi=-\beta$, at that time

$$
\begin{aligned}
& \eta(\rho, \vartheta)=\frac{\beta-2 \kappa^{2}}{3}+2 \kappa^{2} \tanh (\kappa(\rho-\xi \vartheta))^{2}, \varpi(\rho, \vartheta)=\xi_{0}+\xi_{1} \tanh (\kappa(\rho-\xi \vartheta)), \\
& \lambda(\rho, \vartheta)=-\frac{4 \kappa^{2} \xi_{0}\left(\beta+\kappa^{2}\right)}{3 \xi_{1}^{2}}+\frac{4 \kappa^{2}\left(\beta+\kappa^{2}\right)}{3 \xi_{1}} \tanh (\kappa(\rho-\xi \vartheta)),
\end{aligned}
$$

are system of $(1.3)$ when $\alpha=1[5,14]$.

In this study, we will use the HAM which was developed by Liao in [12]. This method contains the auxiliary parameter $\hbar$ which provides us with a simple way to adjust and control the convergence region of solution series for large or small values of $\rho$ and $\vartheta[2,3,8,11,13,15,16]$.

\section{Basic definitions}

Definition 2.1. The R-L fractional integral operator of order $\alpha \geqslant 0$ of a function $f \in \xi_{\mu}, \mu \geqslant-1$, is described as $[7,19]$

$$
\mathrm{D}_{\sigma}^{-\alpha} \eta(\rho, \sigma)=\frac{1}{\Gamma(\alpha)} \int_{0}^{\sigma}(\sigma-\sigma)^{\alpha-1} \eta(\rho, \sigma) \mathrm{d} \sigma, \quad \alpha>0, \quad \sigma>0 .
$$

If $\alpha \geqslant 0, m-1>\alpha \leqslant m, m \in N$ and $A^{m}[a, b]$, then [17]

$$
\begin{gathered}
{ }^{C} D^{\alpha} D^{-\alpha} f(\rho)=f(\rho), \\
{ }_{a} D_{\rho}^{-\alpha} C_{a} D_{\rho}^{\alpha} f(\rho)=f(\rho)-\sum_{k=0}^{m-1} \frac{D^{k} f(a)}{k !}(\rho-a)^{k} .
\end{gathered}
$$

Definition 2.2. The C-D is define as $[4,18,22]$

$$
{ }^{c} D_{\sigma}^{\alpha} f(\rho)=\frac{1}{\Gamma(m-\alpha)} \int_{0}^{\sigma}(\sigma-\sigma)^{m-\alpha-1} f^{(m)}(\sigma) d \sigma, \quad m-1<\alpha \leqslant m, \quad m \in N .
$$

\section{The HAM solution of the GHS-cKdV}

According to the system (1.3), we start the application of the HAM for solving this system using the initial conditions. In this system,

$$
\begin{aligned}
& \eta(\rho, 0)=\frac{\beta-2 \kappa^{2}}{3}+2 \kappa^{2} \tanh (\kappa \rho)^{2}, \\
& \lambda(\rho, 0)=-\frac{4 \kappa^{2} \xi_{0}\left(\beta+\kappa^{2}\right)}{3 \xi_{1}^{2}}+\frac{4 \kappa^{2}\left(\beta+\kappa^{2}\right)}{3 \xi_{1}} \tanh (\kappa \rho), \\
& \varpi(\rho, 0)=\xi_{0}+\xi_{1} \tanh (\kappa \rho),
\end{aligned}
$$

are the initial conditions of $\eta(\rho, \vartheta), \lambda(\rho, \vartheta)$ and $\varpi(\rho, \vartheta)[5,14]$. We choose 


$$
\mathrm{L}\left[\psi_{i}(\rho, \vartheta ; p)\right]={ }^{\mathrm{C}} \mathrm{D}_{\vartheta}^{\alpha}\left[\psi_{i}(\rho, \vartheta ; p)\right], \quad i=1,2,3,
$$

with property $L\left[c_{i}\right]=0$, where $c_{i}$ is constants. We define $N_{i}$ by

$$
\begin{aligned}
\mathrm{N}_{1}\left[\psi_{1}(\rho, \vartheta, p)\right]= & { }^{C} D_{\vartheta}^{\alpha} \psi_{1}(\rho, \vartheta ; p)-\frac{1}{2} \frac{\partial^{3} \psi_{1}(\rho, \vartheta ; p)}{\partial \rho^{3}}+3 \psi_{1}(\rho, \vartheta ; p) \frac{\partial \psi_{1}(\rho, \vartheta ; p)}{\partial \rho} \\
& -3 \psi_{2}(\rho, \vartheta ; p) \frac{\partial \psi_{3}(\rho, \vartheta ; p)}{\partial \rho}-3 \frac{\partial \psi_{2}(\rho, \vartheta ; p)}{\partial \rho} \psi_{3}(\rho, \vartheta ; p) \\
N_{2}\left[\psi_{1}(\rho, \vartheta, p)\right]= & { }^{C} D_{\vartheta}^{\alpha} \psi_{2}(\rho, \vartheta ; p)+\frac{\partial^{3} \psi_{2}(\rho, \vartheta ; p)}{\partial \rho^{3}}-3 \psi_{1}(\rho, \vartheta ; p) \frac{\partial \psi_{2}(\rho, \vartheta ; p)}{\partial \rho} \\
\mathrm{N}_{3}\left[\psi_{1}(\rho, \vartheta, p)\right]= & { }^{C} D_{\vartheta}^{\alpha} \psi_{3}(\rho, \vartheta ; p)+\frac{\partial^{3} \psi_{3}(\rho, \vartheta ; p)}{\partial \rho^{3}}-3 \psi_{1}(\rho, \vartheta ; p) \frac{\partial \psi_{3}(\rho, \vartheta ; p)}{\partial \rho}
\end{aligned}
$$

So, we install the zeroth-order deformation equations

$$
\begin{aligned}
& (1-p) \operatorname{L}\left[\psi_{1}(\rho, \vartheta, p)-\eta_{0}(\rho, \vartheta)\right]=p \hbar H(\vartheta) \mathrm{N}\left[\psi_{1}(\rho, \vartheta ; p)\right], \\
& (1-p) \mathrm{L}\left[\psi_{2}(\rho, \vartheta, p)-\lambda_{0}(\rho, \vartheta)\right]=p \hbar H(\vartheta) \mathrm{N}\left[\psi_{2}(\rho, \vartheta ; p)\right] \text {, } \\
& (1-p) \mathrm{L}\left[\psi_{3}(\rho, \vartheta, p)-\varpi_{0}(\rho, \vartheta)\right]=\operatorname{p\hbar H}(\vartheta) \mathrm{N}\left[\psi_{3}(\rho, \vartheta ; p)\right] .
\end{aligned}
$$

In (3.2) for $p=0$ and $p=1$ respectively

$$
\begin{aligned}
& \psi_{1}(\rho, \vartheta, 0)=\eta(\rho, \vartheta), \psi_{1}(\rho, \vartheta, 1)=\eta(\rho, \vartheta), \\
& \psi_{2}(\rho, \vartheta, 0)=\lambda(\rho, \vartheta), \psi_{2}(\rho, \vartheta, 1)=\lambda(\rho, \vartheta), \\
& \psi_{3}(\rho, \vartheta, 0)=\varpi(\rho, \vartheta), \psi_{3}(\rho, \vartheta, 1)=\varpi(\rho, \vartheta) .
\end{aligned}
$$

So we get mth-order DEqs;

$$
\begin{aligned}
\mathrm{L}\left[\eta_{m}(\rho, \vartheta)-\chi_{m} \eta_{m-1}(\rho, \vartheta)\right] & =\hbar H(\vartheta) R_{m}\left(\eta_{m-1}(\rho, \vartheta)\right), \\
L\left[\lambda_{m}(\rho, \vartheta)-\chi_{m} \lambda_{m-1}(\rho, \vartheta)\right] & =\hbar H(\vartheta) R_{m}\left(\lambda_{m-1}(\rho, \vartheta)\right), \\
L\left[\varpi_{m}(\rho, \vartheta)-\chi_{m} \varpi_{m-1}(\rho, \vartheta)\right] & =\hbar H(\vartheta) R_{m}\left(\varpi_{m-1}(\rho, \vartheta)\right) .
\end{aligned}
$$

Here,

$$
\begin{aligned}
& R_{m}\left(\eta_{m-1}(\rho, \vartheta)\right)={ }^{c} D_{\vartheta}^{\alpha} \eta_{m-1}(\rho, \vartheta)-\frac{1}{2} \frac{\partial^{3} \eta_{m-1}}{\partial \rho^{3}}+3 \sum_{i=0}^{m-1} \eta_{i}(\rho, \vartheta) \frac{\partial \eta_{m-1-i}(\rho, \vartheta}{\partial \rho} \\
&-3 \sum_{i=0}^{m-1}\left(\lambda_{i}(\rho, \vartheta) \frac{\partial \varpi_{m-1-i}(\rho, \vartheta)}{\partial \rho}+\varpi_{i}(\rho, \vartheta) \frac{\partial \lambda_{m-1-i}(\rho, \vartheta)}{\partial \rho}\right), \\
& R_{m}\left(\lambda_{m-1}(\rho, \vartheta)\right)={ }^{c} D_{\vartheta}^{\alpha} \lambda_{m-1}(\rho, \vartheta)+\frac{\partial^{3} \lambda_{m-1}(\rho, \vartheta)}{\partial \rho^{3}}-3 \sum_{i=0}^{m-1} \eta_{i}(\rho, \vartheta) \frac{\partial \lambda_{m-1-i}(\rho, \vartheta)}{\partial \rho}, \\
& R_{m}\left(\varpi_{m-1}(\rho, \vartheta)\right)={ }^{c} D_{\vartheta}^{\alpha} \varpi_{m-1}(\rho, \vartheta)+\frac{\partial^{3} \varpi_{m-1}(\rho, \vartheta)}{\partial \rho^{3}}-3 \sum_{i=0}^{m-1} \eta_{i}(\rho, \vartheta) \frac{\partial \varpi_{m-1-i}(\rho, \vartheta)}{\partial \rho} .
\end{aligned}
$$

Now the solution of the mth-order DEqs. (3.3) for $m \geqslant 1$ becomes

$$
\begin{aligned}
\eta_{m}(\rho, \vartheta) & =\chi \eta_{m-1}(\rho, \vartheta)+\hbar H(\vartheta) L^{-1}\left[R_{m}\left(\eta_{m-1}(\rho, \vartheta)\right)\right] \\
\lambda_{m}(\rho, \vartheta) & =\chi \lambda_{m-1}(\rho, \vartheta)+\hbar H(\vartheta) L^{-1}\left[R_{m}\left(\lambda_{m-1}(\rho, \vartheta)\right)\right] \\
\varpi_{m}(\rho, \vartheta) & =\chi \varpi_{m-1}(\rho, \vartheta)+\hbar H(\vartheta) L^{-1}\left[R_{m}\left(\varpi_{m-1}(\rho, \vartheta)\right)\right]
\end{aligned}
$$


If we use (2.1) in Eqs. (3.5), then we get

$$
\begin{aligned}
& \eta_{m}(\rho, \vartheta)=D_{\vartheta}^{-\alpha} C^{c} D_{\vartheta}^{\alpha} \eta(\rho, \vartheta)=\eta(\rho, \vartheta)-\sum_{k=0}^{n-1} \frac{\partial^{k} \eta(\rho, 0)}{\partial \vartheta^{k}} \frac{\vartheta^{k}}{k !}, \vartheta>0, n-1<\alpha<n, \\
& \lambda_{m}(\rho, \vartheta)=D_{\vartheta}^{-\alpha} C^{c} D_{\vartheta}^{\alpha} \lambda(\rho, \vartheta)=\lambda(\rho, \vartheta)-\sum_{k=0}^{n-1} \frac{\partial^{k} \lambda(\rho, 0)}{\partial \vartheta^{k}} \frac{\vartheta^{k}}{k !}, \vartheta>0, n-1<\alpha<n, \\
& \varpi_{m}(\rho, \vartheta)=D_{\vartheta}^{-\alpha} C^{c} D_{\vartheta}^{\alpha} \varpi(\rho, \vartheta)=\varpi(\rho, \vartheta)-\sum_{k=0}^{n-1} \frac{\partial^{k} \varpi(\rho, 0)}{\partial \vartheta^{k}} \frac{\vartheta^{k}}{k !}, \vartheta>0, n-1<\alpha<n .
\end{aligned}
$$

Instead of (3.6) and $R_{m}\left(\eta_{m-1}\right), R_{m}\left(\lambda_{m-1}\right), R_{m}\left(\bigotimes_{m-1}\right)$

$$
\begin{aligned}
\eta_{m}(\rho, \vartheta)= & \left(x_{m}+\hbar\right) \eta_{m-1}(\rho, \vartheta)-\hbar \eta_{m-1}(\rho, 0)+\hbar \frac{1}{\Gamma(\alpha)} \int_{0}^{\vartheta}(\vartheta-\tau)^{\alpha-1} \\
& \times\left(-\frac{1}{2} \frac{\partial^{3} \eta_{m-1}(\rho, \tau)}{\partial \rho^{3}}+3 \sum_{i=0}^{m-1} \eta_{i}(\rho, \tau) \frac{\partial \eta_{m-1-i}}{\partial \rho}-3 \sum_{i=0}^{m-1}\left(\lambda_{i}(\rho, \tau) \frac{\partial \varpi_{m-1-i}}{\partial \rho}\right.\right. \\
& \left.\left.+\varpi_{i}(\rho, \tau) \frac{\partial \lambda_{m-1-i}(\rho, \tau)}{\partial \rho}\right)\right) d \tau \\
\lambda_{m}(\rho, \vartheta)= & \left(x_{m}+\hbar\right) \lambda_{m-1}(\rho, \vartheta)-\hbar \lambda_{m-1}(\rho, 0)+\hbar \frac{1}{\Gamma(\alpha)} \int_{0}^{\vartheta}(\vartheta-\tau)^{\alpha-1} \\
& \times\left(\frac{\partial^{3} \lambda_{m-1}(\rho, \tau)}{\partial \rho^{3}}-3 \sum_{i=0}^{m-1} \eta_{i}(\rho, \tau) \frac{\partial \lambda_{m-1-i}(\rho, \tau)}{\partial \rho}\right) d \tau \\
\varpi_{m}(\rho, \vartheta)= & \left(x_{m}+\hbar\right) \varpi_{m-1}(\rho, \vartheta)-\hbar \varpi_{m-1}(\rho, 0)+\hbar \frac{1}{\Gamma(\alpha)} \int_{0}^{\vartheta}(\vartheta-\tau)^{\alpha-1} \\
& \times\left(\frac{\partial^{3} \varpi_{m-1}(\rho, \tau)}{\partial \rho^{3}}-3 \sum_{i=0}^{m-1} \eta_{i}(\rho, \tau) \frac{\partial \varpi_{m-1-i}(\rho, \tau)}{\partial \rho}\right) d \tau
\end{aligned}
$$

writable and $H(\vartheta)$ can be chosen in the form $H(\vartheta)=1$.

Arrangement of (3.7) gives $\mathrm{m}$-th order deformation equations

$$
\begin{aligned}
\eta_{m}(\rho, \vartheta) & =\chi_{m} \eta_{m-1}(\rho, \vartheta)+\hbar D_{\vartheta}^{-\alpha}\left[R_{m}\left(\eta_{m-1}(\rho, \vartheta)\right)\right], \\
\lambda_{m}(\rho, \vartheta) & =\chi_{m} \lambda_{m-1}(\rho, \vartheta)+\hbar D_{\vartheta}^{-\alpha}\left[R_{m}\left(\lambda_{m-1}(\rho, \vartheta)\right)\right], \\
\varpi_{m}(\rho, \vartheta) & =\chi_{m} \varpi_{m-1}(\rho, \vartheta)+\hbar D_{\vartheta}^{-\alpha}\left[R_{m}\left(\varpi_{m-1}(\rho, \vartheta)\right)\right] .
\end{aligned}
$$

Therefore, we are obtained components as

$$
\begin{aligned}
\eta_{0}(\rho, \vartheta) & =\frac{\beta-2 \kappa^{2}}{3}+2 \kappa^{2} \tanh ^{2}(\kappa \rho), \\
\eta_{1}(\rho, \vartheta) & =-4 \hbar \kappa^{3} \beta \operatorname{sech}^{2}(\kappa \rho) \tanh (\kappa \rho) \frac{\vartheta^{\alpha}}{\Gamma(\alpha+1)}, \\
\eta_{2}(\rho, \vartheta) & =-4 \hbar(1+\hbar) \kappa^{3} \beta \operatorname{sech}^{2}(\kappa \rho) \tanh (\kappa \rho) \frac{\vartheta^{\alpha}}{\Gamma(\alpha+1)}-4^{1-\alpha \hbar^{2} \kappa^{4} \sqrt{\pi} \beta^{2},} \\
& \operatorname{sech}^{4}(\kappa \rho)(-2+\cosh (2 \kappa \rho)) \frac{\vartheta^{2 \alpha}}{\Gamma(\alpha+1) \Gamma\left(\alpha+\frac{1}{2}\right)^{\prime}},
\end{aligned}
$$




$$
\begin{aligned}
& \lambda_{0}(\rho, \vartheta)=-\frac{4 \kappa^{2} \xi_{0}\left(\beta+\kappa^{2}\right)}{3 \xi_{1}^{2}}+\frac{4 \kappa^{2}\left(\beta+\kappa^{2}\right) \tanh (\kappa \rho)}{3 \xi_{1}}, \\
& \lambda_{1}(\rho, \vartheta)=-\frac{4 \hbar \kappa^{3} \beta\left(\beta+\kappa^{2}\right) \operatorname{sech}^{2}(\kappa \rho)}{3 \xi_{1}^{2}} \frac{\vartheta^{\alpha}}{\Gamma(\alpha+1)}, \\
& \lambda_{2}(\rho, \vartheta)=-\frac{4 \hbar(1+\hbar) \kappa^{3} \beta\left(\beta+\kappa^{2}\right) \operatorname{sech}^{2}(\kappa \rho)}{3 \xi_{1}^{2}} \frac{\vartheta^{\alpha}}{\Gamma(\alpha+1)}-8 \hbar^{2} \kappa^{4} \beta^{2}\left(\beta+\kappa^{2}\right), \\
& \left(4^{-\alpha} \sqrt{\left.\operatorname{pi} \vartheta^{2 \alpha} \Gamma(\alpha)\right) \sec ^{2}(\kappa \rho) \tanh (\kappa \rho)},\right. \\
& \vdots \\
& \varpi_{0}(\rho, \vartheta)=\xi_{0}+\xi_{1} \tanh (\kappa \rho), \\
& \varpi_{1}(\rho, \vartheta)=-\hbar \kappa \beta \sec h^{2}(\kappa \rho) \xi_{1} \frac{\vartheta^{\alpha}}{\Gamma(\alpha+1)}, \\
& \varpi_{2}(\rho, \vartheta)=-\hbar(1+\hbar) \kappa \beta \sec h^{2}(\kappa \rho) \xi_{1} \frac{\vartheta^{\alpha}}{\Gamma(\alpha+1)}-2 \hbar^{2} \kappa^{2} \beta^{2} 4^{-\alpha} \sqrt{\pi} \sec ^{2}(\kappa \rho), \\
& \xi_{1} \tanh (\kappa \rho) \frac{\vartheta^{2 \alpha}}{\Gamma(\alpha+1) \Gamma\left(\alpha+\frac{1}{2}\right)^{\prime}}
\end{aligned}
$$

and so on.

As a result, the $m$-th order approximations of $\eta(\rho, \vartheta), \lambda(\rho, \vartheta)$ and $\varpi(\rho, \vartheta)$ are given by

$$
\eta(\rho, \vartheta)=\sum_{m=0}^{\infty} \eta_{m}(\rho, \vartheta), \lambda(\rho, \vartheta)=\sum_{m=0}^{\infty} \lambda_{m}(\rho, \vartheta), \varpi(\rho, \vartheta)=\sum_{m=0}^{\infty} \varpi_{m}(\rho, \vartheta) .
$$

Theorem 3.1 (Convergence Theorem). On conditions that $\eta(\rho, \vartheta)=\eta_{0}(\rho, \vartheta)+\sum_{m=1}^{\infty} \eta_{m}(\rho, \vartheta), \lambda(\rho, \vartheta)=$ $\lambda_{0}(\rho, \vartheta)+\sum_{m=1}^{\infty} \lambda_{m}(\rho, \vartheta)$ and $\varpi(\rho, \vartheta)=\varpi_{0}(\rho, \vartheta)+\sum_{m=1}^{\infty} \varpi_{m}(\rho, \vartheta)$ converges, where $\eta_{m}(\rho, \vartheta), \lambda_{m}(\rho, \vartheta)$ and $\varpi_{m}(\rho, \vartheta)$ are governed by (3.8) under the definitions (3.4) and (3.5), it must be solutions of time-fraction GHS-cKdV equation (1.3).

Proof. If the series

$$
\sum_{m=0}^{\infty} \eta_{m}(\rho, \vartheta), \quad \sum_{m=0}^{\infty} \lambda_{m}(\rho, \vartheta), \quad \sum_{m=0}^{\infty} \varpi_{m}(\rho, \vartheta),
$$

convergence, then we can write

$$
\begin{aligned}
& S_{1}(\rho, \vartheta)=\sum_{m=0}^{\infty} \eta_{m}(\rho, \vartheta), \\
& S_{2}(\rho, \vartheta)=\sum_{m=0}^{\infty} \lambda_{m}(\rho, \vartheta), \\
& S_{3}(\rho, \vartheta)=\sum_{m=0}^{\infty} \varpi_{m}(\rho, \vartheta),
\end{aligned}
$$

and it holds

$$
\begin{aligned}
& \lim _{n \rightarrow \infty} \eta_{n}(\rho, \vartheta)=0, \\
& \lim _{n \rightarrow \infty} \lambda_{n}(\rho, \vartheta)=0,
\end{aligned}
$$




$$
\lim _{n \rightarrow \infty} \varpi_{n}(\rho, \vartheta)=0
$$

By using the definition (3.4), we get

$$
\begin{aligned}
\hbar \sum_{m=1}^{\infty} R_{m}\left(\eta_{m-1}(\rho, \vartheta)\right) & =\sum_{m=1}^{\infty} L\left[\eta_{m}(\rho, \vartheta)-\chi_{m} \eta_{m-1}(\rho, \vartheta)\right] \\
& =\lim _{n \rightarrow \infty} \sum_{m=1}^{\infty} L\left[\eta_{m}(\rho, \vartheta)-\chi_{m} \eta_{m-1}(\rho, \vartheta)\right] \\
& =L\left[\lim _{n \rightarrow \infty} \sum_{m=1}^{\infty}\left(\eta_{m}(\rho, \vartheta)-\chi_{m} \eta_{m-1}(\rho, \vartheta)\right)\right] \\
& =L\left[\lim _{n \rightarrow \infty} \eta_{n}(\rho, \vartheta)\right]=0 .
\end{aligned}
$$

Similarly,

$$
\begin{aligned}
& \hbar \sum_{m=1}^{\infty} R_{m}\left(\lambda_{m-1}(\rho, \vartheta)\right)=L\left[\lim _{n \rightarrow \infty} \lambda_{n}(\rho, \vartheta)\right]=0, \\
& \hbar \sum_{m=1}^{\infty} R_{m}\left(\varpi_{m-1}(\rho, \vartheta)\right)=L\left[\lim _{n \rightarrow \infty} \varpi_{n}(\rho, \vartheta)\right]=0 .
\end{aligned}
$$

From $\hbar \neq 0, \sum_{m=1}^{\infty} R_{m}\left(\eta_{m}^{\rightarrow}(\rho, \vartheta)\right)=0, \sum_{m=1}^{\infty} R_{m}\left(\lambda_{m}(\rho, \vartheta)\right)=0$ and $\sum_{m=1}^{\infty} R_{m}\left(\varpi_{m}^{\rightarrow}(\rho, \vartheta)\right)=0$. From (3.4), it holds

$$
\begin{aligned}
\sum_{m=1}^{\infty} R_{m} & \left(\eta_{m-1}(\rho, \vartheta)\right) \\
= & \sum_{m=1}^{\infty}\left[{ }^{C} D_{\vartheta}^{\alpha} \eta_{m-1}(\rho, \vartheta)-\frac{1}{2}\left(\eta_{m-1}(\rho, \vartheta)\right) \rho \rho \rho+3 \sum_{i=0}^{m-1}\left(\eta_{i}(\rho, \vartheta) \eta_{m-1-i}(\rho, \vartheta)\right)_{\rho}\right. \\
& -\lambda_{i}(\rho, \vartheta) \varpi_{m-1-i}(\rho, \vartheta)-\varpi_{i}(\rho, \vartheta) \lambda_{m-1-i}(\rho, \vartheta) \\
= & \sum_{m=1}^{\infty} D_{\vartheta}^{\alpha} \eta_{m-1}(\rho, \vartheta)-\frac{1}{2} \sum_{m=1}^{\infty}\left(\eta_{m-1}(\rho, \vartheta)\right) \rho \rho \rho+3 \sum_{m=1}^{\infty}\left[\sum _ { i = 0 } ^ { m - 1 } \left(\eta_{i}(\rho, \vartheta)\left(\eta_{m-1-i}(\rho, \vartheta)_{\rho}\right)\right.\right. \\
& \left.-\lambda_{i}(\rho, \vartheta)\left(\varpi_{m-1-i}(\rho, \vartheta)\right)_{\rho}-\varpi_{i}(\rho, \vartheta)\left(\lambda_{m-1-i}(\rho, \vartheta)\right)_{\rho}\right] \\
= & \left.\sum_{m=0}^{\infty} D_{\vartheta}^{\alpha} \eta_{m}(\rho, \vartheta)\right)-\frac{1}{2} \sum_{m=0}^{\infty}\left(\eta_{m}(\rho, \vartheta)\right) \rho \rho \rho+3 \sum_{i=0}^{\infty} \sum_{m=i+1}^{\infty}\left[\left(\eta_{i}(\rho, \vartheta)\left(\eta_{m}-1-i(\rho, \vartheta)_{\rho}\right)\right]\right. \\
& \left.-\lambda_{i}(\rho, \vartheta)\left(\varpi_{m}-1-i(\rho, \vartheta)\right)_{\rho}-\varpi_{i}(\rho, \vartheta)\left(\lambda_{m}-1-i(\rho, \vartheta)\right)_{\rho}\right] \\
= & D_{\vartheta}^{\alpha} \sum_{m=0}^{\infty} \eta_{m}(\rho, \vartheta)-\frac{1}{2}\left(\sum_{m=0}^{\infty} \eta_{m}(\rho, \vartheta)\right)_{\rho \rho \rho}+3\left(\sum_{i=0}^{\infty} \eta_{i}(\rho, \vartheta) \sum_{m=i+1}^{\infty}\left(\eta_{m-1-i}(\rho, \vartheta)\right)_{\rho}\right. \\
& \left.-\sum_{i=0}^{\infty} \lambda_{i}(\rho, \vartheta) \sum_{m=i}^{\infty}\left(\varpi_{m-1}(\rho, \vartheta)\right)_{\rho}-\sum_{i=0}^{\infty} \varpi_{i}(\rho, \vartheta) \sum_{m=i+1}^{\infty}\left(\lambda_{m-1-i}(\rho, \vartheta)\right)_{\rho}\right) \\
= & D_{\vartheta}^{\alpha} S_{1}(\rho, \vartheta)-\frac{1}{2}\left(S_{1}(\rho, \vartheta)\right)_{\rho \rho \rho}+3\left(S_{1}(\rho, \vartheta)\left(S_{1}(\rho, \vartheta)\right)_{\rho}-S_{2}(\rho, \vartheta)\left(S_{3}(\rho, \vartheta)\right)_{\rho}\right. \\
& -S_{3}(\rho, \vartheta)\left(S_{2}(\rho, \vartheta)\right)_{\rho} \\
= & 0 .
\end{aligned}
$$

From the condition $\eta(\rho, 0)=0$ and $\eta_{m}(\rho, 0)=0$,

$$
S_{1}(\rho, 0)=\sum_{m=0}^{\infty} \eta_{m}(\rho, 0)=\eta_{0}(\rho, \vartheta)+\sum_{m=1}^{\infty} \eta_{m-1}(\rho, 0)=f(\rho, \vartheta) .
$$


In the same like (3.9)

$$
\sum_{m=1}^{\infty} R_{m}\left(\lambda_{m-1}(\rho, \vartheta)\right)=0, \sum_{m=1}^{\infty} R_{m}\left(\varpi_{m-1}(\rho, \vartheta)\right)=0 .
$$

From the conditions $\lambda(\rho, 0)=0, \lambda_{m}(\rho, 0)=0$ and $\varpi(\rho, 0)=0, \varpi_{m}(\rho, 0)=0$,

$$
\begin{gathered}
S_{2}(\rho, 0)=\sum_{m=0}^{\infty} \lambda_{m}(\rho, 0)=\lambda_{0}(\rho, \vartheta)+\sum_{m=1}^{\infty} \lambda_{m-1}(\rho, 0)=g(\rho, \vartheta), \\
S_{3}(\rho, 0)=\sum_{m=0}^{\infty} \varpi_{m}(\rho, 0)=\varpi_{0}(\rho, \vartheta)+\sum_{m=1}^{\infty} \varpi_{m-1}(\rho, 0)=h(\rho, \vartheta) .
\end{gathered}
$$

Consequently, $S(\rho, \vartheta)$ must be the exact solution of (1.3) and (3.1).

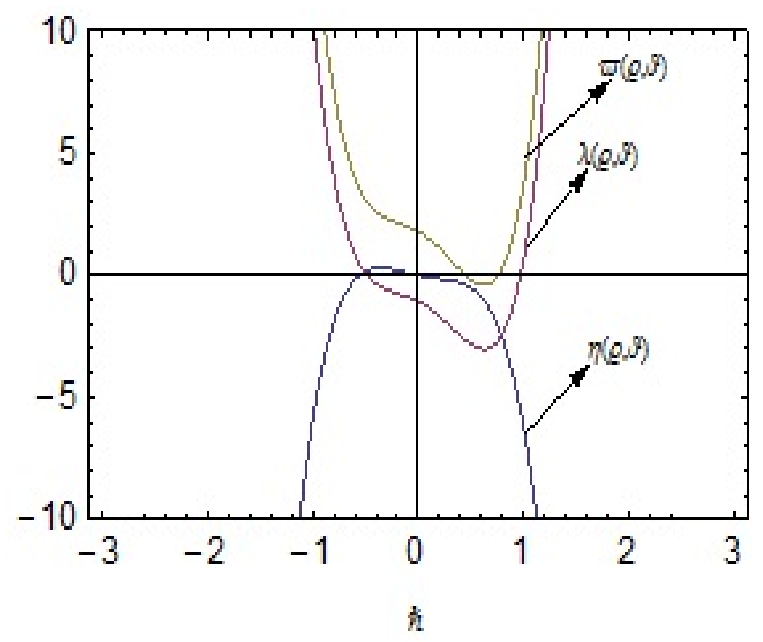

Figure 1: The $\hbar$ curves of 5 th-order approximate solutions obtained by the HAM for $\alpha=0.5$ and $\kappa=1, \beta=0.5, \xi_{0}=\xi_{1}=$ $1.5, \rho=0.2, \vartheta=0.6$.

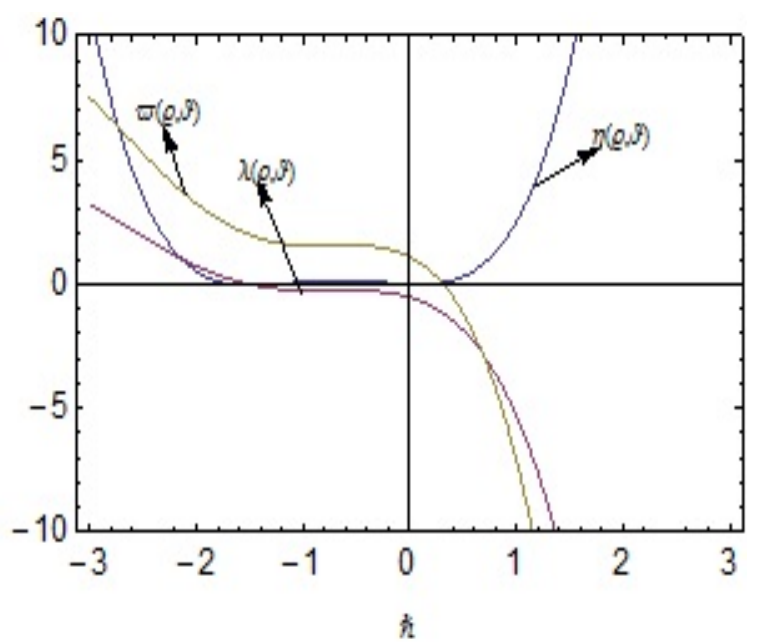

Figure 2: The $\hbar$ curves of 5 th-order approximate solutions obtained by the HAM for $\alpha=0.75$ and $\kappa=0.5, \beta=1.5, \xi_{0}=\xi_{1}=$ $1, \rho=0.2, \vartheta=0.6$. 
Table 1: Absolute errors obtained when $\alpha=0.5$ and $\kappa=0.1, \beta=1.5, \rho=0.5$ for 3th-order approximation of $\eta(\rho, \vartheta)$.

\begin{tabular}{|c|c|c|c|c|}
\hline$\hbar$ & $\vartheta$ & DTM[9] & HPM[6] & HAM \\
\hline-2.6 & 0.2 & 0.00121759 & 0.00142938 & $5.77383 \mathrm{E}-4$ \\
\hline-2.2 & 0.4 & 0.00157146 & 0.00188767 & $5.10103 \mathrm{E}-4$ \\
\hline-2.35 & 0.6 & 0.00193597 & 0.002317283 & $5.55 \mathrm{E}-6$ \\
\hline-2.2 & 0.8 & 0.00230311 & 0.00271511 & $4.0728 \mathrm{E}-7$ \\
\hline
\end{tabular}

Table 2: Absolute errors obtained when $\alpha=0.5$ and $\kappa=0.1, \beta=1.5, \xi_{0}=\xi_{1}=1.5, \rho=0.5$ for 3th-order approximation of $\lambda(\rho, \vartheta)$.

\begin{tabular}{|c|c|c|c|c|}
\hline$\hbar$ & $\vartheta$ & DTM[9] & HPM[6] & HAM \\
\hline-1.75 & 0.2 & $2.66655 \mathrm{E}-4$ & $2.66655 \mathrm{E}-4$ & $2.4006 \mathrm{E}-5$ \\
\hline-1.651 & 0.4 & $5.40902 \mathrm{E}-4$ & $4.22079 \mathrm{E}-4$ & $6.95901 \mathrm{E}-6$ \\
\hline-1.54 & 0.6 & $6.212223 \mathrm{E}-4$ & $5.06039 \mathrm{E}-4$ & $4.2746 \mathrm{E}-6$ \\
\hline-1.45 & 0.8 & $4.13316 \mathrm{E}-4$ & $5.33157 \mathrm{E}-4$ & $2.36771 \mathrm{E}-5$ \\
\hline
\end{tabular}

Table 3: Absolute errors obtained when $\alpha=0.5$ and $\kappa=0.1, \beta=1.5, \xi_{0}=\xi_{1}=1.5, \rho=0.5$ for 3th-order approximation of $\varpi(\rho, \vartheta)$.

\begin{tabular}{|c|c|c|c|c|}
\hline$\hbar$ & $\vartheta$ & DTM[9] & HPM[6] & HAM \\
\hline-2.22 & 0.2 & 0.0677777 & 0.02998 & $1.35667 \mathrm{E}-4$ \\
\hline-0.336 & 0.4 & 0.0694248 & 0.0471694 & $1.28267 \mathrm{E}-7$ \\
\hline-0.438 & 0.6 & 0.0604485 & 0.0565524 & $1.21137 \mathrm{E}-4$ \\
\hline-0.54 & 0.8 & 0.0461901 & 0.059583 & $1.6585 \mathrm{E}-4$ \\
\hline
\end{tabular}

Table 4: HAM, ES and absolute errors (AE) results of $\eta(\rho, \vartheta)$ when $\kappa=1, \beta=0.5, \xi_{0}=\xi_{1}=1.5, \rho=0.25$ and $\alpha=0.5$ for 5th-order approximation.

\begin{tabular}{|l|l|l|l|l|}
\hline$\hbar$ & $\vartheta$ & HAM & ES & AE \\
\hline-0.9 & 0.1 & 0.0826045 & 0.0826252 & $2.07372 \mathrm{E}-5$ \\
\hline-0.388 & 0.2 & 0.172619 & 0.1722751 & $1.31932 \mathrm{E}-4$ \\
\hline-0.416 & 0.3 & 0.259719 & 0.259898 & $1.79292 \mathrm{E}-4$ \\
\hline-0.42 & 0.4 & 0.343514 & 0.343798 & $2.84257 \mathrm{E}-4$ \\
\hline-0.417 & 0.5 & 0.424265 & 0.424234 & $3.03379 \mathrm{E}-5$ \\
\hline-0.411 & 0.6 & 0.501284 & 0.50104 & $2.43098 \mathrm{E}-4$ \\
\hline-0.404 & 0.7 & 0.574846 & 0.574099 & $7.46711 \mathrm{E}-4$ \\
\hline-0.396 & 0.8 & 0.643616 & 0.64334 & $2.75958 \mathrm{E}-4$ \\
\hline-0.388 & 0.9 & 0.70864 & 0.708736 & $9.58075 \mathrm{E}-5$ \\
\hline-0.381 & 1.0 & 0.77238 & 0.770298 & $2.08196 \mathrm{E}-5$ \\
\hline
\end{tabular}

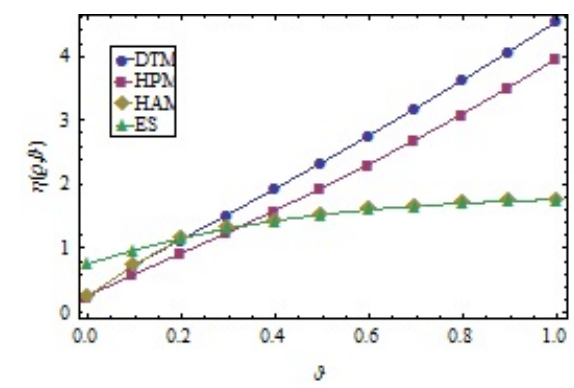

Figure 3: DTM, HPM, HAM and ES. for 3th-order approximate when $\alpha=0.75, \kappa=1, \beta=1.5$ and $\rho=0.5$ of $\eta(\rho, \vartheta)$. 
Table 5: HAM, ES and absolute errors (AE) results of $\lambda(\rho, \vartheta)$ when $\kappa=0.1, \beta=1.5, \xi_{0}=\xi_{1}=1, \rho=0.25$ and $\alpha=0.5$ for 5th-order approximation.

\begin{tabular}{|l|l|l|l|l|}
\hline$\hbar$ & $\vartheta$ & HAM & ES & AE \\
\hline-0.079 & 0.1 & -0.0193281 & -0.0193284 & $3.09595 \mathrm{E}-7$ \\
\hline-0.12 & 0.2 & -0.0190208 & -0.0190271 & $6.29367 \mathrm{E}-6$ \\
\hline-0.153 & 0.3 & -0.0187258 & -0.0187263 & $4.907072 \mathrm{E}-7$ \\
\hline-0.185 & 0.4 & -0.0184285 & -0.0184261 & $2.39303 \mathrm{E}-6$ \\
\hline-0.218 & 0.5 & -0.0181262 & -0.0181267 & $5.32422 \mathrm{E}-7$ \\
\hline-0.25 & 0.6 & -0.0178328 & -0.0178282 & $4.62445 \mathrm{E}-6$ \\
\hline-0.285 & 0.7 & -0.0175349 & -0.0175306 & $4.26116 \mathrm{E}-6$ \\
\hline-0.324 & 0.8 & -0.0172347 & -0.0172343 & $4.4655 \mathrm{E}-7$ \\
\hline-0.37 & 0.9 & -0.0169306 & -0.0169392 & $8.63545 \mathrm{E}-6$ \\
\hline-0.419 & 1.0 & -0.0166457 & -0.0166455 & $1.75463 \mathrm{E}-7$ \\
\hline
\end{tabular}

Table 6: HAM, ES and absolute errors (AE) results of $\varpi(\rho, \vartheta)$ when $\kappa=0.1, \beta=0.5, \xi_{0}=\xi_{1}=0.5, \rho=0.25$ and $\alpha=0.5$ for 5th-order approximation.

\begin{tabular}{|l|l|l|l|l|}
\hline$\hbar$ & $\vartheta$ & HAM & ES & AE \\
\hline-0.079 & 0.1 & 0.514998 & 0.514996 & $2.23619 \mathrm{E}-6$ \\
\hline-0.1187 & 0.2 & 0.517499 & 0.517493 & $2.76808 \mathrm{E}-6$ \\
\hline-0.153 & 0.3 & 0.519989 & 0.519989 & $2.1848 \mathrm{E}-7$ \\
\hline-0.1859 & 0.4 & 0.522491 & 0.522485 & $6.301298 \mathrm{E}-7$ \\
\hline-0.218 & 0.5 & 0.52497 & 0.524979 & $9.60925 \mathrm{E}-6$ \\
\hline-0.2516 & 0.6 & 0.527472 & 0.527472 & $4.41146 \mathrm{E}-6$ \\
\hline-0.287 & 0.7 & 0.52997 & 0.529964 & $6.24215 \mathrm{E}-6$ \\
\hline-0.325 & 0.8 & 0.532452 & 0.532454 & $2.527737 \mathrm{E}-6$ \\
\hline-0.3685 & 0.9 & 0.534951 & 0.534943 & $8.18447 \mathrm{E}-6$ \\
\hline-0.419 & 1.0 & 0.537423 & 0.53743 & $6.6887 \mathrm{E}-6$ \\
\hline
\end{tabular}

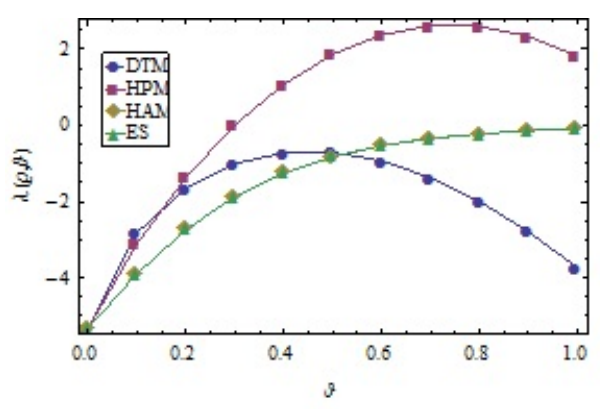

Figure 4: DTM, HPM, HAM and ES. for 3th-order approximate when $\alpha=0.75, \mathrm{~K}=1.5, \beta=1.5, \xi_{0}=\xi_{1}=1.5$ and $\rho=0.2$ of $\lambda(\rho, \vartheta)$.

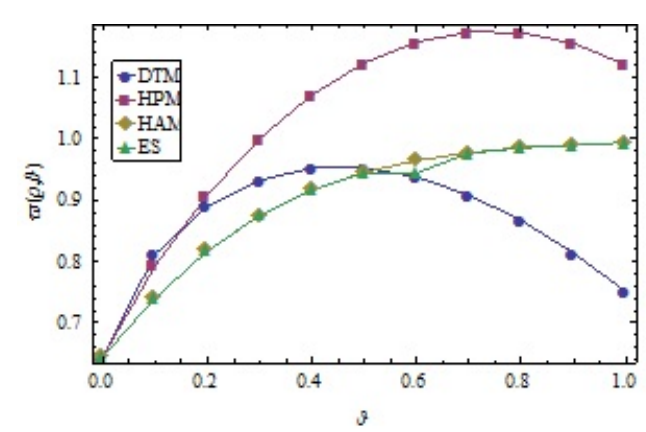

Figure 5: DTM, HPM, HAM and ES. for 3th-order approximate when $\alpha=0.75, \mathrm{~K}=1.5, \beta=1.5, \xi_{0}=\xi_{1}=0.5$ and $\rho=0.2$ of $\varpi(\rho, \vartheta)$.

\section{Conclusion}

In this article, we have achieved approximate solutions of a time-fraction GHS-cKdV equation via the HAM.

We have made convergence analysis and the range of $\hbar \neq 0$ was determined in Figures 1 and 2 . Absolute errors that were obtained by DTM [14] , HPM [5] and HAM have been seen in Tables 1-3. As it has been seen from these tables, when $\hbar$ takes selecting the approximate, HAM gave better results than the other two methods. In Tables 4-6, a comparison between HAM, ES and absolute error that was 
obtained by HAM for 5-terms of $\eta, \lambda$ and $₫$ was showed. In Figures 3-5, a comparison between DTM, HPM, HAM and ES for $\alpha=0.75$ and some values of $\kappa, \beta, \xi_{0}, \xi_{1}$ and convergence control parameter $\hbar$ was presented. As it has been shown from the figures, DTM and HPM solutions walked off from ES but HAM solution approached the ES.

Consequently, in spite of solution which is found by HAM observed very closed to analytical solution, it is shown that solutions which are obtained with DTM and HPM are far to analytical solutions. In conclusion, HAM provides us with a simple way to adjust and control the convergence regions and rates of approximation solution.

\section{References}

[1] R. Abazari, M. Abazari, Numerical simulation of generalized Hirota-Satsuma coupled KdV equation by RDTM and comparison with DTM, Commun. Nonlinear Sci. Numer. Simul., 17 (2012), 619-629. 1, 1

[2] S. Abbasbandy, Soliton solutions for the Fitzhugh-Nagumo equation with the homotopy analysis method, Appl. Math. Model., 32 (2008), 2706-2714. 1

[3] S. Abbasbandy, E. Shivanian, Series solution of the system of integro-differential equations, Z. Naturforsch. A, 64 (2009), 811-818. 1

[4] D. Baleanu, O. P. Agrawal, Fractional Hamilton formalism within Caputo's derivative, Czechoslovak J. Phys., 56 (2006), 1087-1092. 2.2

[5] Z. Z. Ganji, D. D. Ganji, Y. Rostamiyan, Solitary wave solutions for a time-fraction generalized Hirota-Satsuma coupled KdV equation by an analytical technique, Appl. Math. Model., 33 (2009), 3107-3113. 1, 3, 4

[6] C. S. Gardner, J. M. Greene, M. D. Kruskal, R. M. Miura, Korteweg-deVries equation and generalization. VI. Methods for exact solution, Comm. Pure Appl. Math., 27 (1974), 97-133. 1, 1, 2, 3

[7] A. K. Golmankhaneh, A. Golmankhaneh, D. Baleanu, On nonlinear fractional Klein-Gordon equation, Signal Process., 91 (2011), 446-451. 2.1

[8] A. K. Golmankhaneh, N. A. Porghoveh, D. Baleanu, Mean square solutions of second-order random differential equations by using homotopy analysis method, Rom. Rep. Phys., 65 (2013), 350-362. 1

[9] R. Hirota, Exact solution of the Korteweg—de Vries equation for multiple collisions of solitons, Phys. Rev. Lett., 27 (1971), 1192-1194. 1, 1, 2, 3

[10] R. Hirota, J. Satsuma, Soliton solutions of a coupled Korteweg-de Vries equation, Phys. Lett. A, 85 (1981), 407-408. 1

[11] M. Inc, On exact solution of Laplace equation with Dirichlet and Neumann boundary conditions by the homotopy analysis method, Phys. Lett. A, 365 (2007), 412-415. 1

[12] S.-J. Liao, Beyond perturbation, Introduction to the homotopy analysis method, CRC Series: Modern Mechanics and Mathematics, Chapman \& Hall/CRC, Boca Raton, FL, (2003). 1

[13] Y.-P. Liu, Z.-B. Li, The homotopy analysis method for approximating the solution of the modified Korteweg-de Vries equation, Chaos Solitons Fractals, 39 (2009), 1-8. 1

[14] J.-C. Liu, H. Li, Approximate analytic solutions of time-fractional Hirota-Satsuma coupled KdV equation and coupled MKdV equation, Abstr. Appl. Anal., 2013 (2013), 11 pages. 1, 3, 4

[15] H. Jafari, S. Das, H. Tajadodi, Solving a multi-order fractional differential equation using homotopy analysis method, J. King Saud Univ. Sci., 23 (2011), 151-155. 1

[16] H. Jafari, S. Seifi, Homotopy analysis method for solving linear and nonlinear fractional diffusion-wave equation, Commun. Nonlinear Sci. Numer. Simul., 14 (2009), 2006-2012. 1

[17] S. Momani, Z. Odibat, A. Alawneh, Variational iteration method for solving the space-and time-fractional KdV equation, Numer. Methods Partial Differential Equations, 24 (2008), 262-271. 2.1

[18] I. Podlubny, Fractional differential equations, An introduction to fractional derivatives, fractional differential equations, to methods of their solution and some of their applications, Mathematics in Science and Engineering, Academic Press, Inc., San Diego, CA, (1999). 2.2

[19] M. Shateri, D. D. Ganji, Solitary wave solutions for a time-fraction generalized Hirota-Satsuma coupled KdV equation by a new analytical technique, Int. J. Differ. Equ., 2010 (2010), 10 pages. 2.1

[20] X.-J. Yang, Advanced Local Fractional Calculus and Its Applications, World Sci., NewYork, USA, (2012). 1

[21] X.-J. Yang, D. Baleanu, Y. Khan, S. T. Mohyud-Din, Local fractional variational iteration method for diffusion and wave equations on Cantor sets, Romanian J. Phys., 59 (2014), 36-48. 1

[22] X.-J. Yang, D. Baleanu, H. M. Srivastava, Local fractional integral transforms and their applications, Elsevier/Academic Press, Amsterdam, (2016). 2.2

[23] X.-J. Yang, H. M. Srivastava, J.-H. He, D. Baleanu, Cantor-type cylindrical-coordinate method for differential equations with local fractional derivatives, Phys. Lett. A, 377 (2013), 1696-1700. 1 